


\section{Proceedings of the 38th International Symposium on Automation and Robotics in Construction}

ISSN (for the proceedings series): 2413-5844

ISBN (for this issue of the proceeding series): 978-952-69524-1-3

The proceedings series is Scopus indexed.

\section{Scopus}

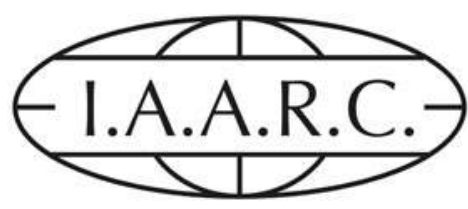

Copyright reserved.

(c) 2021 International Association on Automation and Robotics in Construction

This work including all its parts is protected by copyright. Any use outside the narrow limits of copyright law without the consent of the individual authors is inadmissible and punishable. This applies in particular to reproductions, translations, microfilming and saving and processing in electronic systems.

The reproduction of common names, trade names, trade names etc. in this work does not justify the assumption that such names are to be regarded as free within the meaning of the trademark and trademark protection legislation and can therefore be used by everyone, even without special identification.

Cover design: Thomas Linner, Rongbo Hu, Annika Hartz; Image: Facade Processing Robot commissioned by the Construction Industry Council Hong Kong. 


\section{Editorial Board}

\section{Editors in Chief}

Feng, Chen | Linner, Thomas | Brilakis, Ioannis

\section{Editors (Area Chairs)}

Castro, Daniel

Chen, Po-Han

Cho, Yong

Du, Jing

Ergan, Semiha

Garcia de Soto, Borja

Gašparík, Jozef

Habbal, Firas

Hammad, Amin

Iturralde, Kepa \& Bock, Thomas

Kwon, Soonwook

Lafhaj, Zoubeir

Li, Nan

Liang, Ci-Jyun
Mantha, Bharadwaj

Ng, Ming Shan \& Hall, Daniel

Pan, Mi \& Pan, Wei

Rahimian, Farzad

Raphael, Benny

Sattineni, Anoop

Schlette, Christian

Shabtai, Isaac

Shen, Xuesong

Tang, Pingbo

Teizer, Jochen

Turkan, Yelda

Valero, Enrique

Zhu, Zhenhua 


\section{Outstanding Reviewers}

Alizadehsalehi, Sepehr

Barati, Khalegh

Chen, Jiawei

Chen, Long

Chi, Hung-Lin

Dorrie, Robin

Getuli, Vito

Hackl, Jürgen

Hartmann, Timo

Jacobsen, Emil Lybaek

Kim, Yeseul

König, Markus

Lachmayer, Lukas

Li, Duanshun

Lin, Jing
Mantha, Bharadwaj

Mossman, Alan

Pal, Aritra

Pianet, Grégoire

Prieto, Samuel A.

Riise, Jonathan

Shi, Yangming

Shi, Ying

Sonkor, Muammer Semih

Sun, Zhe

Tsai, Meng-Han

Wang, Yanyu

Xiao, Yong

Yao, Dongchi

Zheng, Zhenjie 


\section{Local Organizing Committee}

Habbal, Firas

Safi, Ammar

Naqi, Asma
Al Ali, Rafeeah

Habbal, Fawaz 


\section{Foreword}

ISARC is the premiere global conference in the domain of automation and robotics in construction. Specifically, ISARC 2021 is situated in an extremely interesting and important time in history: interest in digitising, automating, and robotising construction is rising in an unprecedented scale on a global level and cross all actors. This manifests itself in an increase in academic research activity, joint industry-academia collaboration projects, the emergence of strong start-ups, and a strong and growing interest of large, established organisations (contractors, machine/robot builders, governments etc.).

To prepare ISARC for the future, this year a first attempt was made to restructure IAARC's topic framework. The technical areas summarize the paper topic areas of interest, which represents all the research themes that are relevant to ISARC/IAARC. This is an important mechanism for the technical committee to consolidate the knowledge accumulated from each year's conference while allowing for the smooth incorporation of new research topics and trends in the community.

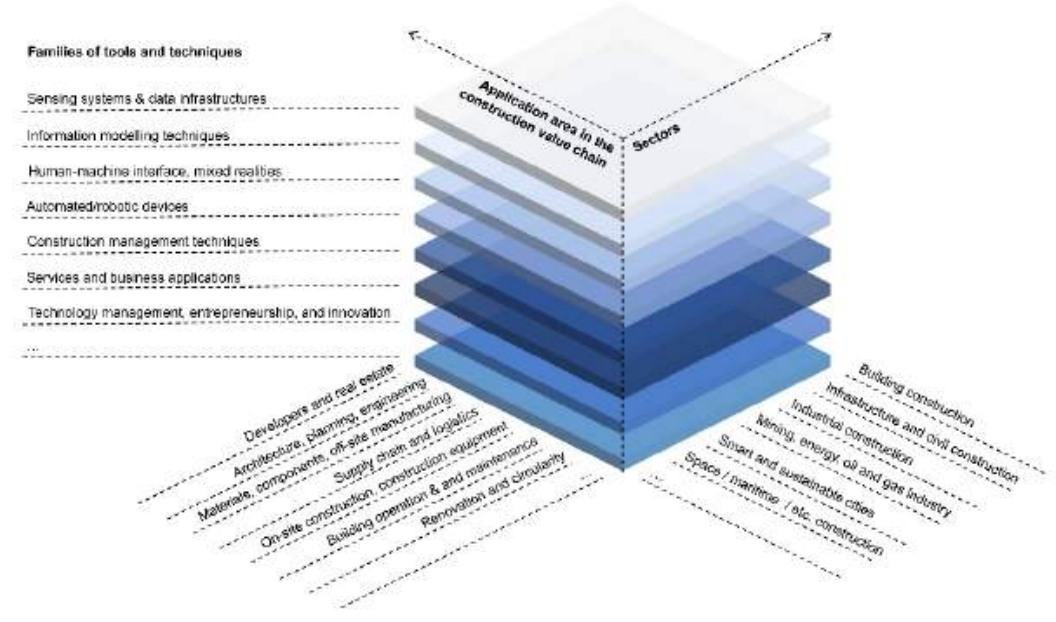

We hope that ISARC 2021 and the proceedings serves the audience and readers and all actors interested in automation and robotics in construction as a navigation and reference system for the domain and its most recent outputs and innovations.

Chen Feng, Thomas Linner, Ioannis Brilakis 


\section{KP Keynote and plenary talks}

Collaborative Welding and Joint Sealing Robots with Haptic Feedback.

Cynthia Brosque, Elena Galbally, Yuxiao Chen, Ruta Joshi, Oussama Khatib and Martin Fischer

Fabrication Information Modeling: Closing the Gap Between Building Information Modeling

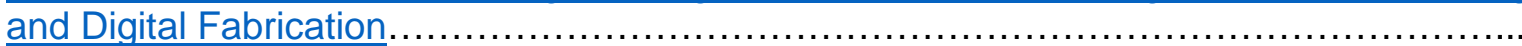
Martin Slepicka, Simon Vilgertshofer and André Borrmann

A Methodology for BIM-enabled Automated Reality Capture in Construction Inspection with Quadruped Robots....

Srijeet Halder, Kereshmeh Afsari, John Serdakowski and Stephen DeVito

iHRC: An AR-based Interface for Intuitive, Interactive and Coordinated Task Sharing

Between Humans and Robots in Building Construction ....

Felix Amtsberg, Xiliu Yang, Lior Skoury, Hans-Jakob Wagner and Achim Menges

A Web-Based GIS Tool for Progress Monitoring of Linear Construction

\section{Projects.}

Vineela Thellakula, Varun Kumar Reja and Koshy Varghese

Towards a Distributed Intelligent Home Based on Smart Furniture for China's Aging Population: A Survey $\ldots \ldots \ldots \ldots \ldots \ldots \ldots \ldots \ldots \ldots \ldots \ldots \ldots \ldots \ldots \ldots \ldots \ldots \ldots \ldots \ldots \ldots \ldots \ldots \ldots \ldots \ldots \ldots \ldots \ldots \ldots$
Rongbo Hu, Thomas Linner, Suting Wang, Wenting Cheng, Xiaolong Liu, Jörg Güttler, Charlie Zhao, Yuan Lu and Thomas Bock

Vision-based Excavator Activity Analysis and Safety Monitoring System Sibo Zhang and Liangjun Zhang

Semantic Optimal Robot Navigation Using Building Information on Construction Sites....... Sina Karimi, Rafael Gomes Braga, Ivanka Iordanova and David St-Onge

CIM-enabled Quantitative View Assessment in Architectural Design and Space

Planning

Vikrom Laovisutthichai, Maosu Li, Fan Xue, Weisheng Lu, K.L. Tam and Anthony G.O. Yeh

\section{A Sensing systems \& data infrastructures}

A Generalizability Analysis of a Data-driven Method for the Urban Heat Island

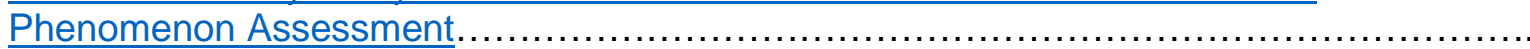
Monica Pena Acosta, Faridaddin Vahdatikhaki, Joao Oliveira dos Santos, Amin Hammad and Andre Doree

A Conceptual Framework for Secure BIM-based Design: Using Blockchain and Asymmetric Encryption....

A Digital Twin Framework for Enhancing Predictive Maintenance of Pumps in Wastewater

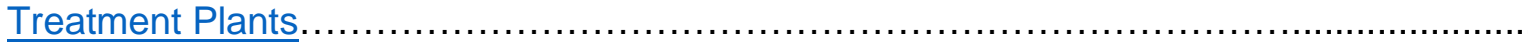
Seyed Mostafa Hallaji, Yihai Fang and Brandon Winfrey 
The BIMERR Interoperability Framework: Towards BIM Enabled Interoperability in the

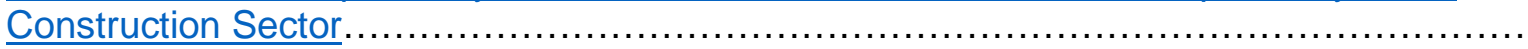

Nefeli Bountouni, Fenareti Lampathaki, Spiros Kousouris, Anastasios Tsitsanis, Georgios Vafeiadis and Danai Vergeti

Use of BIM in the Analysis of Concrete Damage Structures: A Systematic Review of the

Literature.....

Marcella de Sena Barbosa, Francisca Ires Vieira de Melo and Josyanne Pinto Giesta

Towards a Construction Site Control System - Task Management in Construction

Operations and Intralogistics....

Maximilian Schöberl, Daniel Bartmann, Stephan Kessler and Johannes Fottner

Blockchain-based E-tendering Evaluation Framework.

Xingbo Gong, Xingyu Tao, Moumita Das, Yuhan Liu and Jack Cheng

The Needs and Trends of Urban Simulation Platforms- A Review.

Yun-Tsui Chang, Aritra Pal, Jürgen Hackl and Shang-Hsien Hsieh

Towards an Integrated Building Information Modeling (BIM) and Geographic Information

System (GIS) Platform for Infrastructure.

Ashtarout Ammar and Gabriel Dadi

Realizing, Twinning, and Applying IFC-based 4D Construction Management Information

Model of Prefabricated Buildings....

Miaosi Dong, Bin Yang, Boda Liu, Zhichen Wang and Binghan Zhang

Enabling Operational Autonomy in Earth-moving with Real-time3D Environment Modelling. Ross Walker, Simon Smith and Frédéric Bosché

Automated Model Preprocessing for Structural Analysis.

Goran Sibenik, Iva Kovacic and Valentinas Petrinas

Bridge Inspection Field Support and Inspection Method by Heat Map Using 3D Point

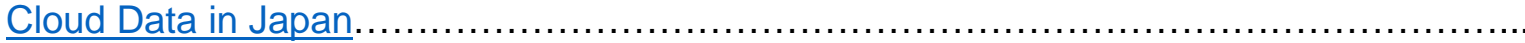

Kazuhiko Seki, Aika Yamaguchi and Satoshi Kubota

Developing a knowledge-based system for semantic enrichment and automatic BIM-based quantity take-off

A BIM Integrated Hazardous Zone Registration Using Image Stitching

Si Tran, Truong Linh Nguyen and Chansik Park

Integration of Building Material Databases for IFC-based Building Performance Analysis... Stefan Fenz, Julia Bergmayr, Nico Plattner, Serge Chávez-Feria, María Poveda-Villalón and Giorgos Giannakis

Dam Sustainability Assessment and Flood Volume Estimation Using Remote Sensing and

GIS.

Saeid Kalantari, Mohammad Ahmadi, Khalegh Barati and Shokoufeh Khojeh

Vision-based Precast Concrete Management Plan in Off-Site Construction Site: Using PC

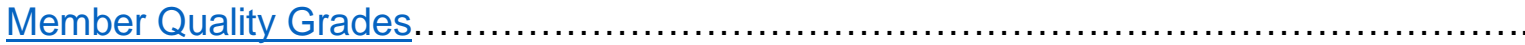
Ziqing Zhu, Yik Pong Yong, Seojoon Lee, Younghee Chang and Soonwook Kwon

An Integrated Scan-to-BIM Approach for Buildings Energy Performance Evaluation and

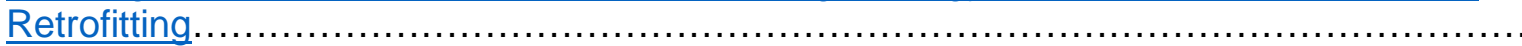

Enrique Valero, Dibya D. Mohanty, Michal Ceklarz, Boan Tao, Frederic Bosche, Giorgos I. Giannakis, Stefan Fenz, Kyriakos

Katsigarakis, Georgios N. Lilis, Dimitrios Rovas and Antonis Papanikolaou 
A Proof-of-concept Application of Sensing Technologies for Managing Proximity Hazards

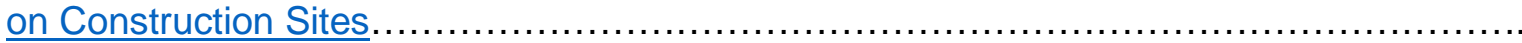
Silvia Mastrolembo Ventura, Paolo Bellagente, Andrea Rossi, Sara Comai, Alessandra Flammini, Stefano Rinaldi and

\section{B Information modeling techniques}

A Systematic Review of Automated BIM Modelling for Existing Buildings from 2D

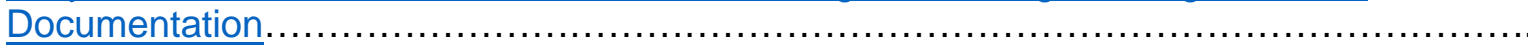

Cheng Zhang, Yang Zou and Johannes Dimyadi

Enhancing Deep Learning-based BIM Element Classification via Data Augmentation and

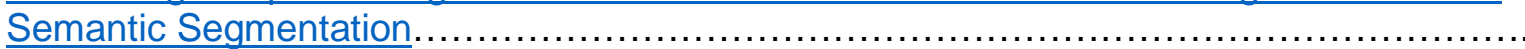

Youngsu Yu, Koeun Lee, Daemok Ha and Bonsang Koo

Design Optimization Approach Comparing Multicriterial Variants Using BIM in Early

Design Stages ...

Kasimir Forth, Jimmy Abualdenien, André Borrmann, Sabrina Fellermann and Christian Schunicht

Establish a Cost Estimation Model for Pre-sold Home Customization Based on BIM and

VR

Sheng-Han Tung, Kun-Chi Wang and Ping-Yu Yu

Towards Near Real-time Digital Twins of Construction Sites: Developing High-LOD 4D

Simulation Based on Computer Vision and RTLS.

Yusheng Huang, Amin Hammad, Ghazaleh Torabi, Ali Ghelmani and Michel Guevremont

Logging Modeling Events to Enhance the Reproducibility of a Modeling Process.....

Towards HLA-based Modeling of Interdependent Infrastructure Systems

Joseph Jonathan Magoua, Fei Wang and Nan Li

From the Graphical Representation to the Smart Contract Language: A Use Case in the

Construction Industry

Xuling Ye and Markus König

Case Study

Steven Chuo, Qian Chen, Mohammad Mahdi Sharif, Carl T. Haas and Bryan T. Adey

Formulation of Construction Equipment Replacement and Retrofitting Strategies for

Emission Reduction

Zhenhua Huang and Hongqin Fan

Spatio-temporal Planning Simulation of Temporary Elevators in Construction Using a

Game Engine

Keyi Wu and Borja García de Soto

Discrete Event Simulation of Multi-purpose Utility Tunnels Construction Using

Microtunneling.....

Shayan Jorjam and Amin Hammad

Importance of Secondary Processes in Heavy Equipment Resource Scheduling Using

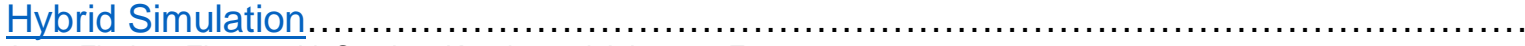

Anne Fischer, Zhuoran Li, Stephan Kessler and Johannes Fottner

Develop and Benchmark FDS Numerical Models to Simulate Fundamental Fire Behavior 
Towards Intelligent Agents to Assist in Modular Construction: Evaluation of Datasets

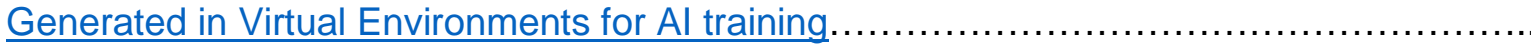

Keundeok Park, Semiha Ergan and Chen Feng

Using Image Processing to Classify Roofs based on Damage Level.

Fast Online Incremental Segmentation of 3D Point Clouds from Disaster Sites....

Yosuke Yajima, Seongyong Kim, Jing Dao Chen and Yong Cho

Vision-Based Progress Monitoring of Building Structures Using Point-Intensity Approach...

Varun Kumar Reja, Parth Bhadaniya, Koshy Varghese and Quang Phuc Ha

Quantity Estimation of Executed Works Using Image Analytics.

Intelligent Archiving of Interior Design Images using Panorama Picture Sources....

A Timely Object Recognition Method for Construction using the Mask R-CNN Architecture Dena Shamsollahi, Osama Moselhi and Khashayar Khorasani

Point Cloud Semantic Segmentation of Concrete Surface Defects Using Dynamic Graph

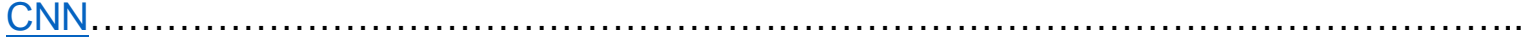

Fardin Bahreini and Amin Hammad

Deep Learning-Based Entity Recognition in Construction Regulatory Documents....

Phillip Schönfelder and Markus König

A Pull-Reporting Approach for Floor Opening Detection Using Deep-Learning on

Embedded Devices.

Sharjeel Anjum, Rabia Khalid, Muhammad Khan, Numan Khan and Chansik Park

Snowplow Route Optimization Using Chinese Postman Problem and Tabu Search

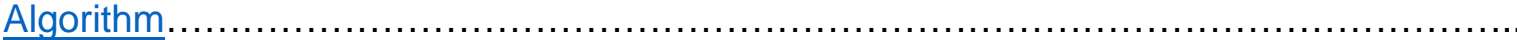

Abdullah Rasul, Jaho Seo, Shuoyan Xu, Tae J. Kwon, Justin MacLean and Cody Brown

A Deep Learning-based Multi-model Ensemble Method for Crack Detection in Concrete

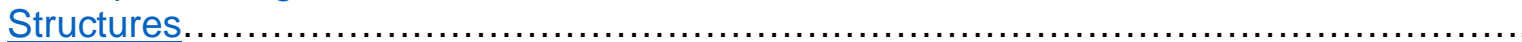

Luqman Ali, Farag Sallabi, Wasif Khan, Fady Alnajjar and Hamad Aljassmi

A Deep Learning-based detection of Fall Portents for Lone Construction Worker.

Numan Khan, Sharjeel Anjum, Rabia Khalid, Junsung Park and Chansik Park

Data Cleaning for Prediction and its Evaluation of Building Energy Consumption.....

Yun-Yi Zhang, Zhen-Zhong Hu, Jia-Rui Lin and Jian-Ping Zhang

BIM-based Variant Retrieval of Building Designs Using Case-based Reasoning and

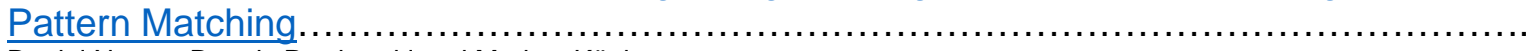

Daniel Napps, Dennis Pawlowski and Markus König

Image Dedusting with Deep Learning based Closed-Loop Network. 


\section{Human factors \& human-system collaboration}

Real-time Digital Twin of Robotic construction processes in Mixed Reality.....

Kaushik Selva Dhanush Ravi, Ming Shan Ng, Jesús Medina Ibáñez and Daniel Mark Hall

Augmented Reality to Increase Efficiency of MEP Construction: A Case Study.....

Patrick Dallasega, Felix Schulze, Andrea Revolti and Martin Martinelli

Markerless Augmented Reality for Facility Management: Automated Spatial Registration

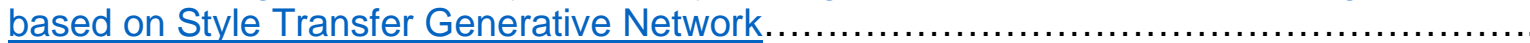

Junjie Chen, Shuai Li, Weisheng Lu, Donghai Liu, Da Hu and Maohong Tang

Remote Monitoring System and Controller for the Construction Machinery using AR Smart

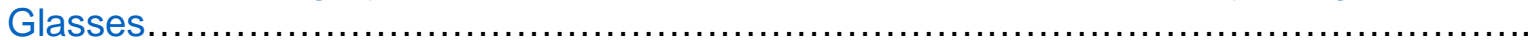
Ali Akbar, Jinwoo Song, Jungtaek Hong, Kyuhyup Lee and Soonwook Kwon

BIM-LCA Integration for Energy Modeling of Modular Residential Projects....

Nassim Mehrvarz, Khalegh Barati and Xuesong Shen

Challenges in Deictic Gesture-Based Spatial Referencing for Human-Robot Interaction in

Construction.....

Sungboo Yoon, Yeseul Kim, Changbum Ahn and Moonseo Park

Wearable Sensor-based Hand Gesture Recognition of Construction Workers

Xin Wang and Zhenhua Zhu

Context-appropriate Social Navigation in Various Density Construction Environment using

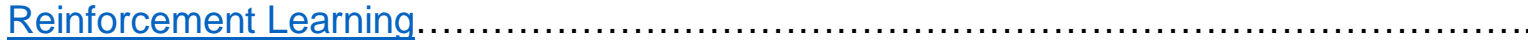

Yeseul Kim, Bogyeong Lee, Robin Murphy and Changbum Ahn

Factors Affecting Inspection Staffing Needs for Highway Construction Projects

Makram Bou Hatoum, Hala Nassereddine, Timothy R.B. Taylor and Steve Waddle

Addressing COVID-19 Spatial Restrictions on Construction Sites Using a BIM-Based

Gaming Environment.

Leonardo Messi, Borja García de Soto, Alessandro Carbonari and Berardo Naticchia

A Dynamic Graph-based Time Series Analysis Framework for On-site Occupational

Hazards Identification

Shi Chen, Feiyan Dong and Kazuyuki Demachi

Is Safety Climate Different by Project Size and Activity with Different Risk Levels?

Hyunho Jung and Youngcheol Kang

Designing an Experiment to Measure the Alert Fatigue of Different Alarm Sounds Using

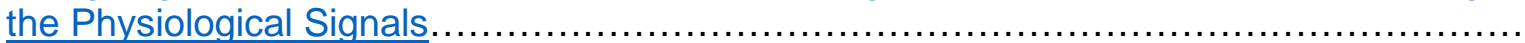

Jeonghyeun Chae and Youngcheol Kang

Adaptive Zoom Control Approach of Load-View Crane Camera for Worker Detection.......... Tanittha Sutjaritvorakul, Atabak Nejadfard, Axel Vierling and Karsten Berns

Application of Game Engine Technology for Construction Safety Training: A Middle

Eastern Case Study.....

Ali Ezzeddine and Hiam Khoury

Evaluation and Comparison of The Performance of Artificial Intelligence Algorithms in

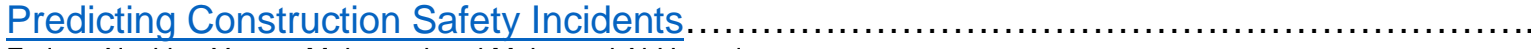

Fatima Alsakka, Yasser Mohamed and Mohamed Al-Hussein 
Estimating Hazard Exposure in Tower Crane Lift Operations Using BIM and Path Planning

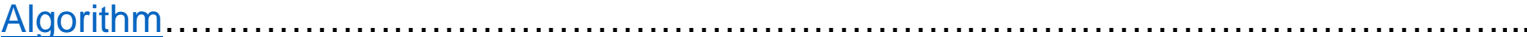

Songbo Hu, Yihai Fang and Robert Moehler

Detecting Hook Attachments of a Safety Harness Using Inertial Measurement Unit

Sensors...

Hoonyong Lee, Namgyun Kim and Changbum Ahn

Semi-automatic Construction Hazard Identification Method Using 4D BIM

Mohammad Saeed Heidary, Milad Mousavi, Amin Alvanchi, Khalegh Barati and Hossein Karimi

Tunneling in Unmanned Construction ...

Takumi Moteki, Ziwei Qiao, Yuichi Mizukoshi and Hiroyasu Iwata

Digitalization-based situation awareness for construction safety management - A critical

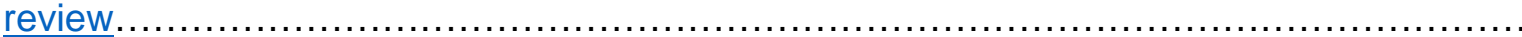

Zhe Zhang, Brian H.W. Guo, Alice Chang-Richards, Ruoyu Jin and Yu Han

Asymmetrical Multiplayer Serious Game and Vibrotactile Haptic Feedback for Safety in Virtual Reality to Demonstrate Construction Worker Exposure to Overhead Crane Loads... Ingvild Moelmen, Haavard L. Grim, Emil Lybaek Jacobsen and Jochen Teizer

A Conceptual Framework for Construction Safety Training using Dynamic Virtual Reality

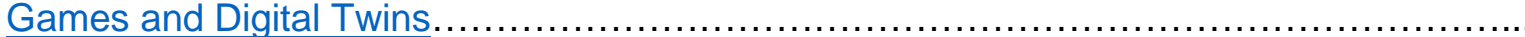
Aparna Harichandran, Karsten W. Johansen, Emil L. Jacobsen and Jochen Teizer

Autonomous Safety Barrier Inspection in Construction: An Approach Using Unmanned Aerial Vehicles and Safe BIM.... Karsten W. Johansen, Rui Pimentel de Figueiredo, Olga Golovina and Jochen Teizer

\section{Robotic machines, devices and end-effectors}

ABECIS: an Automated Building Exterior Crack Inspection System using UAVs, Open-

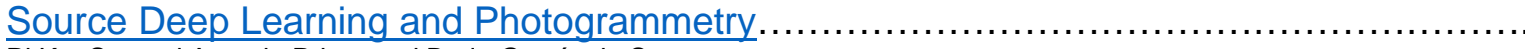

Pi Ko, Samuel Antonio Prieto and Borja García de Soto

Unmanned Aerial System Integration for Monitoring and Management of Landslide: A

Case of Dominican Republic....

Hamlet David Reynoso Vanderhorst

Conceptual Design of a Robotic Building Envelope Assessment System for Energy

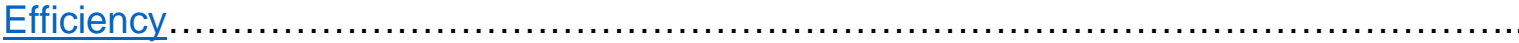

Xuchu Xu, Daniel Lu, Bilal Sher, Sunglyoung Kim, Abhishek Rathod, Semiha Ergan and Chen Feng

Implementation of a Robotic System for Overhead Drilling Operations: A Case Study of

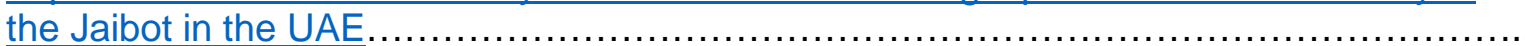

Xinghui Xu, Tyron Holgate, Pinar Coban and Borja García de Soto

AutoCIS: An Automated Construction Inspection System for Quality Inspection of Buildings Samuel A. Prieto, Nikolaos Giakoumidis and Borja García de Soto

Robotics Applicability for Routine Operator Tasks in Power Plant Facilities

Hafiz Oyediran, Prashnna Ghimire, Matthew Peavy, Kyungki Kim and Philip Barutha

Mechatronic Sliding Formwork Complex Operating Mode Optimization Using its Servos

Technical Condition....

Alexey Bulgakov, Thomas Bock and Tatiana Kruglova 
Simulation-Based Optimization of High-Performance Wheel Loading

Real-time Volume Estimation of Mass in Excavator Bucket with LiDAR Data.....

Extension of an Autopilot Model of Shield Tunneling Machines to Curved Section using

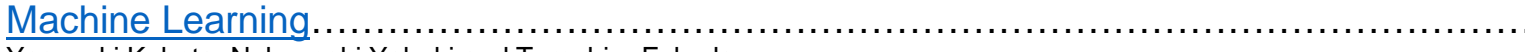

Yasuyuki Kubota, Nobuyoshi Yabuki and Tomohiro Fukuda

Fabrication of a Truss-like Beam Casted with 3D Printed Clay Moulds....

Sébastien Maitenaz, Malo Charrier, Romain Mesnil, Paul Onfroy, Nicolas Metge, Adélaïde Feraille and Jean-François Caron

Automatic Generation of Digital Twin Models for Simulation of Reconfigurable Robotic

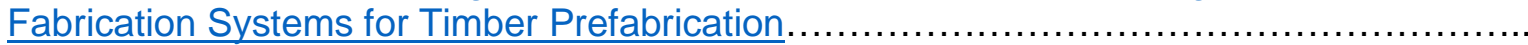

Benjamin Kaiser, Daniel Littfinski and Alexander Verl

Automated shotcrete 3D printing - Printing interruption for extended component

complexity

Lukas Lachmayer, Robin Dörrie, Harald Kloft and Annika Raatz

HAL Robotics Framework.

Tristan Gobin, Sebastian Andraos, Remi Vriet and Thibault Schwartz

Raw Wood Fabrication with Computer Vision

Genki Furusho, Yusuke Nakamura and Gakuhito Hirasawa

Global Localization in Meshes.

Marc Dreher, Hermann Blum, Roland Siegwart and Abel Gawel

Precise Robot Localization in Architectural 3D Plans

Hermann Blum, Julian Stiefel, Cesar Cadena, Roland Siegwart and Abel Gawel

Dynamic Path Generation via Load Monitoring with a Force Sensor for Robot Processing

Using a Chisel....

Yusuke Nakamura and Gakuhito Hirasawa

Image Acquisition Planning for Image-based 3D Reconstruction Using a Robotic Arm....... Rachel Hyo Son and Kevin Han

BIM-integrated Indoor Path Planning Framework for Unmanned Ground Robot.

Zhengyi Chen, Keyu Chen and Jack C. P. Cheng

Semantic Segmentation of 3D Point Cloud Data Acquired from Robot Dog for Scaffold

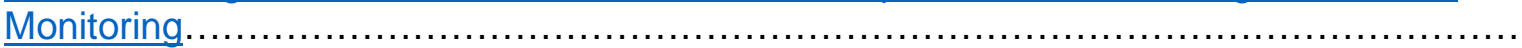

Juhyeon Kim, Duho Chung, Yohan Kim and Hyoungkwan Kim

Modeling and Control of Multi-Units Robotic System: Boom Crane and Robotic Arm

Michele Ambrosino, Philippe Delens and Emanuele Garone

Compilation and Assessment of Automated Façade Renovation....

Kepa Iturralde, Ernesto Gambao and Thomas Bock

Stiffness Study of a Robotic System Working on Vertical Building Surfaces in Construction

Field.....

Elodie Paquet and Benoit Furet 
A Lean-based Production Approach for Shotcrete 3D Printed Concrete Components......... Gerrit Placzek, Leon Brohmann, Karam Mawas, Patrick Schwerdtner, Norman Hack, Mehdi Maboudi and Markus Gerke

Road Map for Implementing Al-driven Oulu Smart Excavator....

Hassan Mehmood, Mikko Hiltunen, Tomi Makkonen, Matti Immonen, Susanna Pirttikangas and Rauno Heikkilä

Hierarchical Planning for Autonomous Excavator on Material Loading Tasks....

Automated Crane-lift Path Planning Using Modified Particle Swarm Optimization for Highrise Modular Integrated Construction.

Aimin Zhu and Wei Pan

Generalization of Construction Object Segmentation Models using Self-Supervised

Learning and Copy-Paste Data Augmentation

Yeji Hong, Wei Chih Chern, Tam Nguyen and Hongjo Kim

\section{E Construction management techniques}

BIM-enabled Sustainability Assessment of Design for Manufacture and Assembly....

Tan Tan, Eleni Papadonikolaki, Grant Mills, Junfei Chen, Zhe Zhang and Ke Chen

A Smart Contract-based BPMN Choreography Execution for Management of Construction

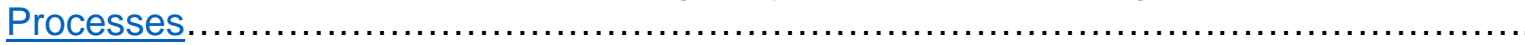
Alessandra Corneli, Francesco Spegni, Marco Alvise Bragadin and Massimo Vaccarini

A Case Based Reasoning Approach for Selecting Appropriate Construction Automation

Method....

Sundararaman Krishnamoorthi and Benny Raphael

Quality Management Processes and Automated Tools for FM-BIM Delivery

Romain Leygonie and Ali Motamedi

An Approach to Data Driven Process Discovery in the Cost Estimation Process of a

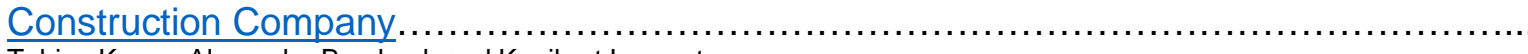

Tobias Kropp, Alexander Bombeck and Kunibert Lennerts

An Ontology Towards BIM-based Guidance of Building Façade Maintenance

From BIM to Inspection: A Comparative Analysis of Assistive Embedment Inspection........ Jeffrey Kim and Darren Olsen

Enhanced Construction Progress Monitoring through Mobile Mapping and As-built 


\section{F Services and business applications}

Note: short papers in this topic are not included in the proceedings.

\section{G Technology management and innovation}

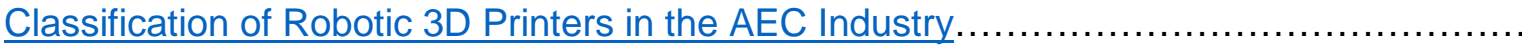

Ala Saif Eldin Sati, Bharadwaj Mantha, Saleh Abu Dabous and Borja García de Soto

Gamification and BIM - The didactic guidance of decentralised interactions of a real-life

BIM business game for higher education.

Christian Heins, Gregor Grunwald and Manfred Helmus

Key Approaches to Construction Circularity: A Systematic Review of The Current State and Future Opportunities...

Qian Chen, Haibo Feng and Borja Garcia de Soto

Key Performance Indicators of Offsite Construction Supply Chains: A Review....

Yidan Zhang, Yi Yang, Wei Pan and Mi Pan

A Proposed Curriculum for Teaching the Tri-Constraint Method of Generative Construction

Scheduling...

Irfan Custovic, Ravina Sriram and Daniel Hall

Understanding and Leveraging BIM Efforts for Electrical Contractors....

Interactions Between Construction 4.0 and Lean Wastes.

Mahmoud El Jazzar and Hala Nassereddine

Application of Digital Twin Technologies in Construction: An Overview of Opportunities

and Challenges...

Haibo Feng, Qian Chen and Borja Garcia de Soto

Analyzing the Impact of Government-driven BIM Adoption: Introducing the Case of South

Korea

Donghoon Ji and Yelda Turkan

Diminished Reality in Architectural and Environmental design: Literature Review of

Techniques, Applications, and Challenges...

Roghieh Eskandari and Ali Motamed

A Qualitative Technology Evaluation Scoreboard for Digital Fabrication in Construction...... Konrad Graser, Jens Hunhevicz, René Jähne, Alexander N. Walzer, Fabian Seiler, Roman Wüst and Daniel M. Hall

Quantifying the Complexity of 3D Printed Concrete Elements...

Smart Contract Using Blockchain in Construction and Infrastructure Sector in the COVID-

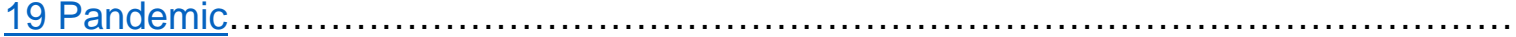

Nawal Alhanaee and Tahani Alhanaee

Towards an Ontology for BIM-Based Robotic Navigation and Inspection Tasks 
Value Stream Mapping of Project Lifecycle Data for Circular Construction....................

Ursula Eicker, Amin Hammad and Osama Moselhi 


\title{
A Proof of Concept Application of Sensing Technologies for Managing Proximity Hazards on Construction Sites
}

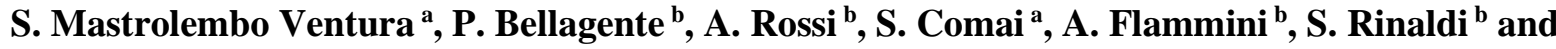 \\ A.L.C. Ciribini ${ }^{\text {a }}$ \\ ${ }^{a}$ Department of Civil, Environmental, Architectural Engineering and Mathematics, University of Brescia, Italy \\ ${ }^{b}$ Department of Information Engineering, University of Brescia, Italy \\ E-mail: silvia.mastrolemboventura@unibs.it, paolo.bellagente@unibs.it, andrea.rossi@ redsandev.com, \\ sara.comai@unibs.it, alessandra.flammini@unibs.it, stefano.rinaldi@unibs.it, angelo.ciribini@unibs.it
}

\begin{abstract}
-
Construction is among the most dangerous industries for the safety of workers. Due to the dynamism typical of construction sites, where workers, materials and equipment resources are often in motion, collisions and contact with moving construction machineries and heavy equipment represents one of the main safety problems. The study described in this paper concerns with the preliminary development of a proximity warning system (PWS) for construction activities, which is based on the implementation of sensing technologies for situation awareness. Preliminary results, the feasibility of the PWS and its practical potential are described, highlighting the needs of a continuous monitoring process and the expectations about the system configuration. The adoption of the Ultra Wide Band (UWB) technology is within the scope of the paper. A front-end loader and an excavator are the construction machineries taken into account for the analysis of the use case, which considers the differences between equipment with fixed and variable geometries in terms of sensor devices. The possibilities for real-time position tracking of workers and equipment in both outdoor and indoor conditions based on the system architecture settings are discussed. Moreover, the compliance of the system architecture with the requirements imposed by the General Data Protection Regulation (GDPR) is described. Future works will validate the system in the context of actual construction sites. Furthermore, factors to be considered when sensing technologies for tracking the position of resources on construction sites are implemented will be evaluated as far as planning and scheduling activities are concerned.
\end{abstract}

Keywords -

Construction safety; Proximity hazards; sensing technologies; Ultra Wide Band; General Data Protection Regulation

\section{Introduction}

Construction represents one of the most dangerous production sectors in terms of risks for the safety of workers [1]. In fact, although around $7 \%$ of the workforce worldwide is employed in construction, this accounts for between $30 \%$ and $40 \%$ of deaths at work [2].

Statistics compiled at international level show that construction safety is a global problem. In the United States, for example, census data from the U.S. Bureau of Labor Statistics (BLS) show that a total of 1.061 workers died in 2019 from fatal work injuries in the construction industry, accounting for $9.7 \%$ of all work-related fatalities; based on these data, construction ranks fourth among all manufacturing sectors for fatal work injuries [3]. As it emerges from the analysis of the data regularly provided by INAIL (Italian National Institute for Insurance against Accidents at Work) in relation to accidents in the workplace, construction has been always considered a high risk sector, with workers exposed to greater risks to their health and safety than in other work environments [4]. Although recording a slight decrease in 2018 compared to previous years, the Italian construction sector also shows the highest rate of accidents causing fatal injuries. In particular, looking at regional data, the accident phenomenon remains concentrated in northern Italy $(60 \%)$, where Lombardy and Emilia Romagna alone account for about a third of all accidents (17\% and $12 \%$ respectively). With regard to the construction sector, albeit with slightly decreasing numbers, in 2018 Lombardy was the region with the most complaints for accidents at work, 1389, followed by Veneto with 1060 ( $-23.7 \%$ complaints compared to Lombardy) and Emilia-Romagna with 982 complaints (29.3\% compared to Lombardy) [4].

1.1 Collision accidents with construction machineries in motion

In this context, proximity of workers to moving 
construction machineries and heavy equipment represents one of the main safety problems in construction. More than six hundred construction worker deaths in the United States between 2004 and 2006 were linked to collisions and contact with moving construction machineries and heavy equipment [5]. INAIL data sets also show that the risk for collision accidents on construction sites represents one of the most frequent causes of death [6]. The causes of this kind of accident could be sought in the dynamic features proper to construction site activities, where the involved resources, such as workers, materials and equipment, are often in motion in situations of excessive proximity on a construction site space that is often insufficient, at least temporarily, to guarantee the safe performance of operations.

Collision accidents, in fact, are often due to a poor analysis of spatial interferences during construction planning and workspace design [7] [8]. Moreover, a lack of knowledge of existing specific risk factors, which is aggravated in daily activities by the possible loss of concentration due to fatigue and repetitive tasks, and the fact that no real-time information is gathered during the incident are some of the main factor influencing collision detection on construction sites [5]. Furthermore, visibility for workers driving construction machineries is often reduced due to blind spots and it increases the probability of risk of workers being run over and invested [2]. In fact, procedural non compliances causing fatal injuries are often related to the non-verification of the absence of operators by the driver of the construction machinery and the positioning of workers in the manoeuvring area. Further issues are detected such as shortcomings in the safety devices of the construction machineries (e.g., rear-view mirrors, reversing horns) and inadequate signposting of pedestrian transit routes [6].

In order to prevent workers being undetected in blind spots or in too close proximity, a warning system is needed that will promptly alert workers and equipment operators [5].

\subsection{Objective of the research project}

The study aimed at the preliminary development of a Proximity Warning System (PWS) for the real-time detection of a potential risk for construction workers because of an excessive proximity to a construction machinery in motion. Such a system has been conceived and developed in the form of a Proof of Concept (PoC) in order to demonstrate, as a preliminary result, its feasibility and practical potential, highlighting the needs of a continuous monitoring process and the expectations of the system configuration.

In particular, sensing technologies are considered in an Internet of Things (IoT) scenario that would allow collecting real-time data directly from the construction site in order to support workers and protect their safety at work, taking into account the balance between safety and productivity. Moreover, the evaluation of the adoption of Ultra Wide Band (UWB) technology as a continuous monitoring system for the safety of construction workers is within the scope of the paper. UWB technology, in fact, has an optimal trade-off between location accuracy and cost of devices, which makes it the ideal solution for sensing precisely the distance in construction sites. Moreover, the design of a precise location service for the PWS should take into consideration workplace privacy, an aspect generally neglected by similar solutions proposed in literature.

In the following paragraphs the study will be introduced with respect to the research background. The research methodology implemented and the system architecture adopted for the development of the proximity warning system will be then described. Furthermore, the compliance of the proximity warning system with the requirements imposed by the General Data Protection Regulation (GDPR) will be shown. Finally, achieved results, findings and outcomes will be discussed in the light of the objectives of the research work, highlighting its limits with respect to the application domain. Possible future developments will be presented in terms of both the use case and the technological system.

\section{Research background}

Although accident prevention is a key step in the management of construction safety, traditional methods such as risk analysis, training of workers, site inspections and compilation of checklists, do not always guarantee optimal levels of continuous safety monitoring and effective and timely prevention of accidents during the construction process. Even when all the aforementioned measures are adopted effectively, workers tend not to recognise as a potential source of risk many of the hazards occurring on the construction site during their own activities [9] [10]; among them, there are the proximity of workers to construction machineries in motion [11].

\subsection{Sensors technologies for situation and context awareness on construction sites}

Strengthening the increasing attention to this type of risk for the safety of workers in the construction sector, the adoption of tracking systems to monitor construction entities (e.g., machineries, workers and materials) is discussed in literature. Vision-based and sensor-based methods exist for this purpose. The former track construction machineries from videos by deploying 
cameras on construction sites. The latter refer to tracking entities by various sensors such as GPS, RFID, UWB, and laser scanners [12].

Cameras have become standard equipment on construction sites [12] However, if currently no visionbased tracking method can achieve processing speed in the tracking of construction machines, especially in the case of multiple ones simultaneously, sensor-based methods for proximity warning systems allows the realtime tracking of construction entities [12]. Moreover, vision systems are also very sensitive to operating conditions (e.g., reduced visibility due to dust on the construction site, or rain) and they require much more maintenance (e.g., frequent cleaning of the system optics). Furthermore, vision-based methods could be more expensive of sensor-based ones (e.g., UWB) and many more cameras are needed to avoid blind spots on the construction site.

Among the sensor-based methods, the use of Inertial Measurement Units (IMU) capable of tracking the kinetic movement data of workers to determine the areas where falls are most likely to occur has been exploited. Other studies have relied on the adoption of Unmanned Aerial Systems (UAS) to identify situations of risk of falling from heights. The increasing use of wearable devices also got the potential to make workers significantly safer on construction sites [13]. The adoption of sensor-based methods has often been referred to proximity warning systems. PWS locate the position of workers in relation to potential safety hazards in the workplace such as proximity to restricted areas and construction machineries in motion. In addition, proximity warning systems use localisation systems not only to monitor the relative position of workers in relation to possible hazards, but also to warn them, by sending notification signals, if they come dangerously close to sources of risk to their safety. Previous studies have shown that the adoption of sensors systems can effectively improve the perception of risks by workers, who are able to take appropriate measures to avoid them once they receive an alert signal [11].

Considering wearable sensor devices, previous studies have used Bluetooth-based Low-Energy (BLE) technology [14] in order to identify the positions of workers with respect to potentially dangerous situations; other attempts, instead, have adopted a real-time localisation system (RTLS) based on Radio-frequency identification (RFID) technology [15] to locate workers and equipment with respect to the position of high-risk or restricted areas, implementing radio-frequency sensing technology also in order to identify possible collisions between workers, heavy equipment and moving construction machineries [5]. Several other technologies, including laser scanning, UWB, Global Positioning System (GPS), and computer vision have been suggested for similar purposes [11].

\subsection{UWB technology}

The adoption of the UWB technology is of particular interest. It has been developed to transmit spectrumdispersive modulated signals over very wide bands (i.e., $500 \mathrm{MHz}$ or more) [16]. UWB applications, in fact, include sensor networks where high data rates are not required but it may be useful to ensure a good coverage range. They also include remote tag localisation systems for applications such as logistics, safety, medical, domestic, security and military.

A typical UWB RTLS is shown in Figure 1. An infrastructure of nodes called anchors is deployed in fixed locations while nodes called tags are applied to the mobile objects which should be tracked. The network coordinator is the node in charge to allow new nodes to join the network as well as to organise the transmission timing among nodes.

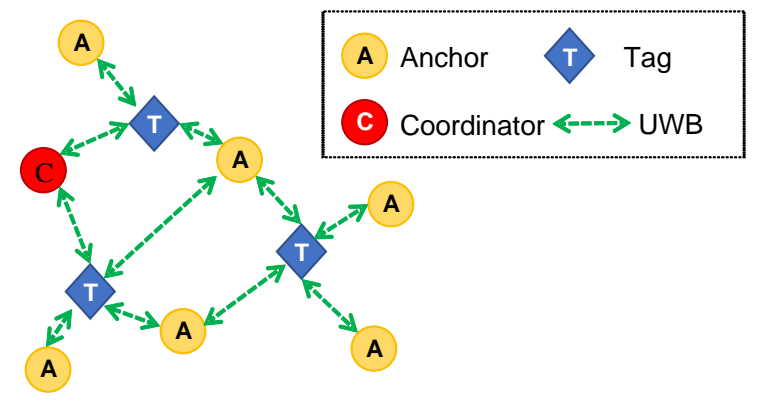

Figure 1 UWB RTLS principle architecture

Usually deployed with a maximum distance of $25 \mathrm{~m}$, anchors send radio pulses to start the location process, which usually has an error within $30 \mathrm{~cm}$ with an update rate every $50 \mathrm{~ms}$, depending on the available hardware. Such a result, it is hard to obtain using GPS technology, unless to adopt solution like GPS receivers with Real Time Kinematic (RTK) support, but which costs are not feasible for a large deployment in construction sites. Usually deployed for automatic asset tracking in indoor environments, UWB technology has several benefits that satisfy large part of the use case requirements. UWB is capable of reaching a range of $100 \mathrm{~m}$ in outdoor environments. It also does not need additional hardware to operate, such as routers. Generally, dynamic scenarios (i.e., with moving objects) such as construction sites can be monitored adopting UWB sensor technologies, but there may be problems if the environment in which the monitoring is done undergoes major changes that prevent, for example, the reception of signals. Moreover, this technology requires manual anchor localisation and registration in RTLS system for tag trilateration. In the literature there are several solutions to address this issue, by using self-configuration approaches, but, the 
complexity, in term of protocol effort, introduced by them is not justified by the considered use case.

\section{Research methodology}

UWB technology was selected for the development of the PWS. Research activities were managed in six macrophases, with some of them overlapping during the testing in order to proceed with an iterative process of data collection and analysis (Figure 2).

- Phase 1 - analysis of the use case for the applicability of the PWS in construction site safety management

- $\quad$ Phase 2 - set up of the system architecture

- Phase 3 - on-site tests for real-time proximity analysis

- $\quad$ Phase 4 - iterative review of the system architecture

- Phase 5 - Verification of the compliance with GPDR

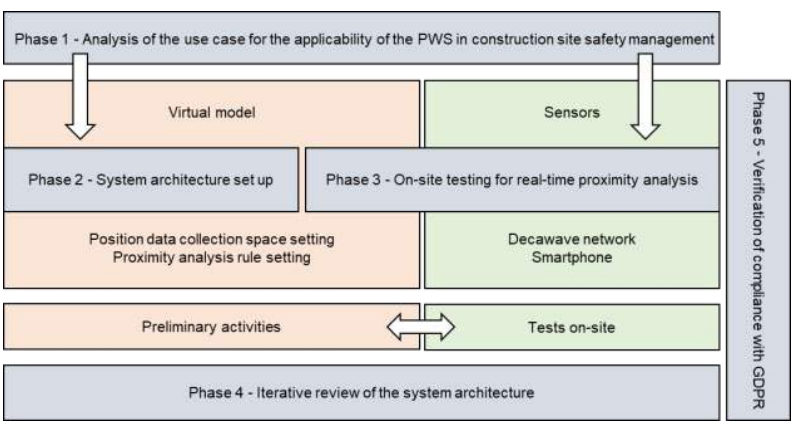

Figure 2 - Research methodology

\subsection{Description of the use case}

The paper analyses the specific use case related to the risk of a construction worker of being run over or invested by a construction machinery in motion. For the purpose of the study, two construction machineries were considered, chosen because of their different configurations: (1) fixed geometry (i.e., the body of the construction machinery has a fixed geometry and configuration during movement) and (2) variable geometry (i.e., the body of the construction machinery has a variable configuration during movement):

1. front-end loader [Takeuchi TL6R] (i.e., fixed geometry) (Figure 3)

2. excavator [Takeuchi TB640] (i.e., variable geometry) (Figure 4) (Figure 5)

Based on technical documentation and workforce experience, five circular areas around the construction machineries have been defined:

1. black area (i.e., the radius of action of the construction machinery) within which there is an imminent permanent injuries or death risk;

2. red area (i.e., an area of $2 \mathrm{~m}$ from the radius of action of the construction machinery in which, according to the manufacturer's instructions, no persons may be present when the equipment is in motion) within which an immediate reaction is needed to avoid permanent injuries or death;

3. yellow area, within which operations are allowed but attention must be made as the risk could quickly increase to red and black;

4. green area, within which the presence of heavy machinery working nearby is notified;

5. white area, within which no risk related to heavy machinery operations is present.

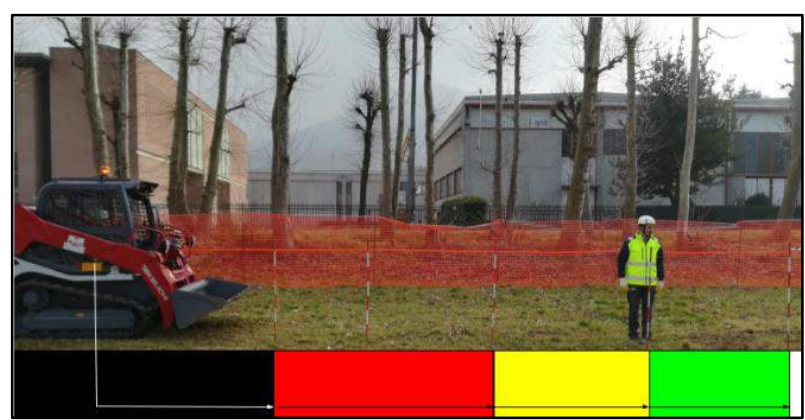

Figure 3 Front-end loader (fixed geometry)

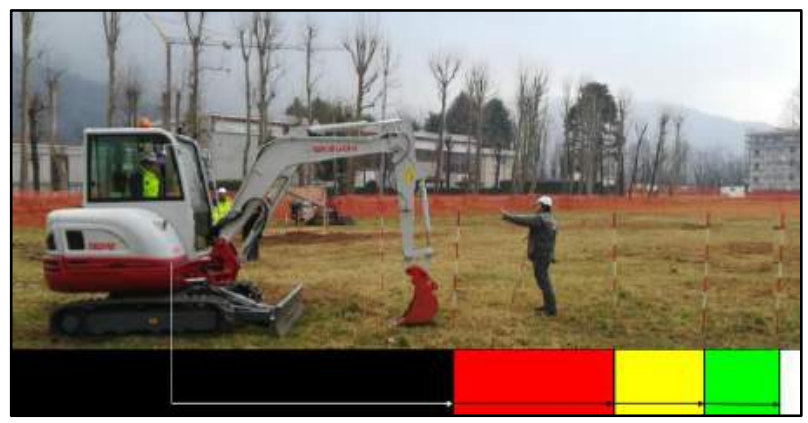

Figure 4 Excavator (variable geometry) retracted arm

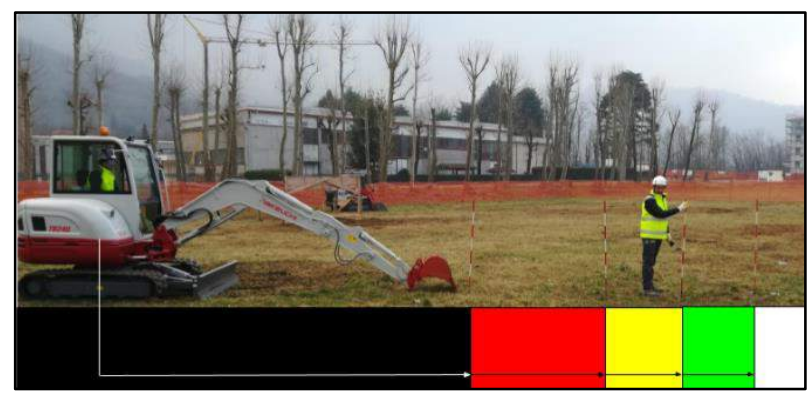

Figure 5 Excavator (variable geometry) extended arm 


\subsection{System architecture setup}

The system architecture setup is shown in Figure 6 and it is composed by three layers: infrastructure, operator and construction machinery.

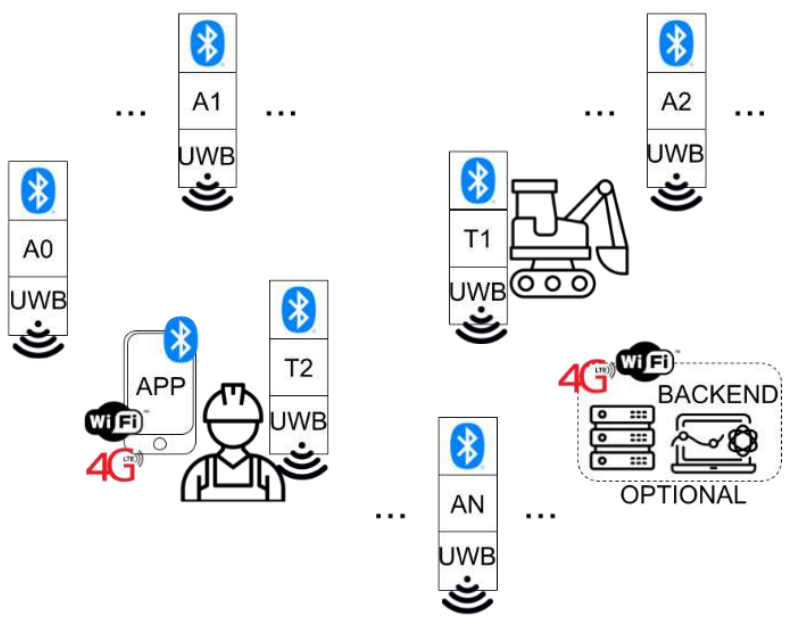

Figure 6 The architecture of UWB position detection system

The infrastructure layer is composed by a network of Decawave DWM1001, which can act as both anchors or tags with a suitable configuration (marked as AX for anchors and TX for tags in Figure 6). Tags are connected to a smartphone, running a custom APP developed for the project, by a Bluetooth Low Energy (BLE) link. The positions of tags are, then, locally collected by the smartphone that could perform the calculations needed to identify the area and alert the operator. If a suitable communication infrastructure (e.g., Wi-Fi or LTE) is available, the smartphone could act as a gateway and forward position data via MQTT (Message Queue Telemetry Transport) to a cloud backend in charge of all the computation. The performance difference between a full cloud or a mixed cloud-edge architecture has been analysed in [17][18].

\subsection{PWS compliance with the General Data Protection Regulation (GDPR) for data privacy}

The development of PWS has raised questions about the risk of violation of the privacy of workers' data due to the continuous monitoring of their position on the construction site to ensure the effective development of an alert system. In this sense, it is underlined that the absolute position of workers is never recorded, while their relative position with respect to a moving construction machinery is recorded: this allows to monitor their exposure to the risk and not their actual location on the construction site.
In addition, each operator has access to data related only to the tags and UWB anchors assigned to a specific worksite. The tag-user association is recorded in the app the PWS is based on and is not exported to other systems; it is also assumed that this association activity is validated by the site manager (e.g., during the delivery of personal protective equipment).

Moreover, each app has a client with a unique ID, which is generated each time the user accesses a specific part of the app. The data saved involves only the tag information regarding the location of the resources and their mutual positioning; if necessary, it is possible to add, as additional data, which client actually sent this location. The tag-media association follows the same logic.

The only information known to all members of the system, therefore, is the type of tag (user/worker, construction machinery) or UWB (which delimits the monitored worksite area). Based on those considerations, the PWS complies, in this preliminary setting, with the European General Data Protection Regulation (GPDR) in terms of data privacy.

\section{Results of the application of sensors technology}

Figure 7 shows the experimental setup. It is formed by 5 anchors (A, B, C, D, E) and a network coordinator I. The area is divided in two part: an indoor area on the left of anchor B and E, and an outdoor area on the right of anchors B and E. B and E are positioned in the indoor side of a concrete wall with a $2 \mathrm{~m}$ open door in the middle (pink line in Figure 7). The experimental set-up has been defined in order to validate the behaviour of UWB sensors in significant operational environments (indoor as well as outdoor).

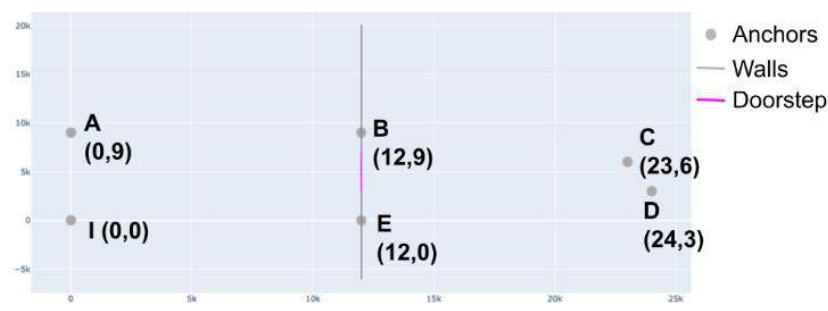

Figure 7 UWB test setup

Data have been collected using the MQTT+ backend configuration leveraging an ad-hoc Wi-Fi network to connect the smartphone with a laptop PC acting as backend. Since the type of movement of the workers and of the construction machinery are different, two set of experiments have been performed, to validate the capability of the UWB technology to monitor each of them independently:

1. detection of an operator equipped with a tag on his chest, as shown in Figure 8 (top); 
2. detection of a moving construction machinery as described in Section 3.1. For the variable geometry of the excavator (i.e., retracted arm and extended arm) two tags have been installed as shown in Figure 8 .

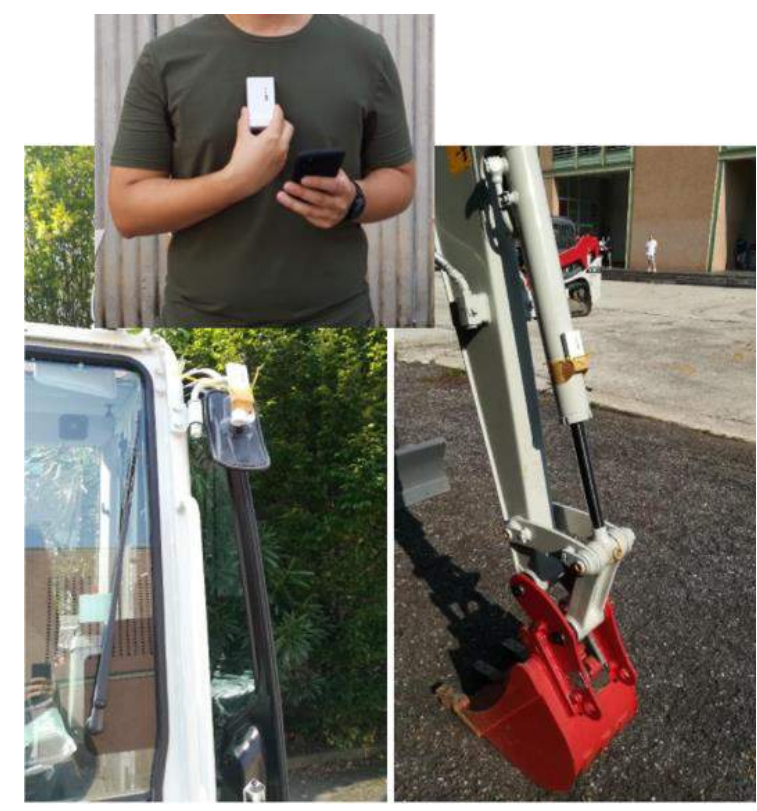

Figure 8 Position of tags for both operator and construction machinery

Figure 9 and Figure 10 show, respectively, the results of the experiments performed during the first and the second set of experiments. In particular, Figure 9 shows the real-time position of a worker walking alone outside and then going inside the building. Figure 10 shows the position of a moving construction machinery, in the same area, without the presence of the worker. The construction machinery started moving in the indoor area. In both the experiments, the position of the workers and of the construction machinery has been sampled each $50 \mathrm{~ms}$.

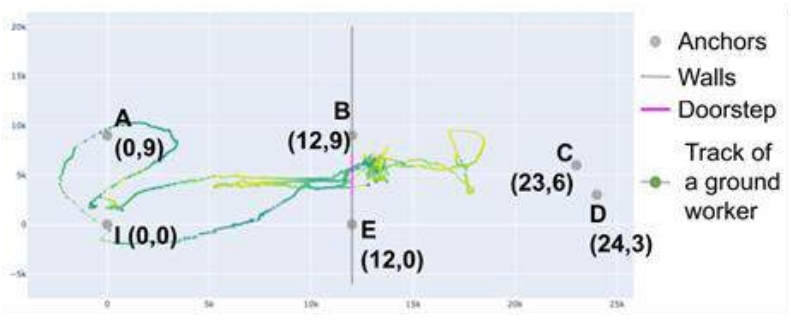

Figure 9 Position tracking of a worker

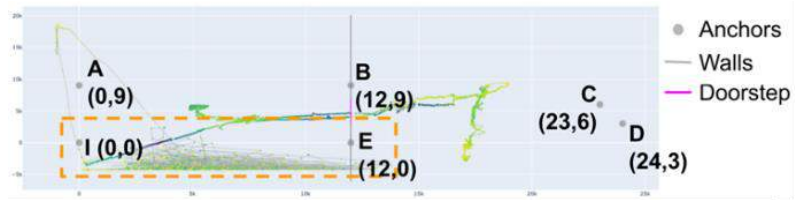

Figure 10 Position tracking of a machinery

Thanks to the location accuracy provided by the UWB sensors (nominal location accuracy of $30 \mathrm{~cm}$ ), the proposed PWS is able to detect the fine details of the movement of the construction machinery. This is the reason of the overlapping trajectories shown in the slashed orange rectangle, which correspond to the operation of the construction machinery.

\section{Conclusions}

\subsection{Discussion of the results}

The obtained results aim to demonstrate the applicability of the proposed system in a near to reality testing environment, which includes the presence of workers as well as construction machinery. As demonstrated by the experimental results, the system is able to track the real-time movement of both a worker as well as of a construction machinery, in indoor as well as outdoor scenarios. With the respect to similar proximity warning solutions used in construction sector based on Bluetooth technology [19] [20] [21] [22], ultrasonic [23] or LoRaWAN devices [24], the proposed solution exploits the use of UWB radio to reach a localization accuracy on the order of $30 \mathrm{~cm}$, at an update rate up to $50 \mathrm{~ms}$, as indicate by the technology provider [25] and by scientific literature Error! Reference source not found..

According to the technology provider documentation [25], there are several factors affecting the localization accuracy. One of the main sources of uncertainty is the power of the received signal, which is affected by the environmental conditions. The UWB technology is only partially affected by multipath effect, because the UWB pulses are transmitted in the RF frequency between $3.5 \mathrm{GHz}$ and $6.5 \mathrm{GHz}$. This frequency band is rather immune to multipath effect, but it could experience the radio frequency attenuation of the signal. According to the device technical document [25], an attenuation of the signal varying in the range from $-60 \mathrm{dbm}$ to $-95 \mathrm{dbm}$, corresponds to a an error in the location estimation from $-20 \mathrm{~cm}$ to $10 \mathrm{~cm}$, respectively.

Notwithstanding these limitations, the UWB remains the most feasible solution for a precise location at a low cost. As demonstrated in the paper, the system is able to track, at the same time, the position of operators and of machineries in the area under monitoring. In fact, when the geometrical distance between a worker and each of 
the construction machineries located in the site is below one of the security areas defined in Section 3.1, an alert or a warning (depending the security area involved) is raised to the involved operator. The location accuracy provided by UWB devices was taken into account when defining the security areas, to avoid any risks for the workers.

In addition, according to the recent trends in proximity warning system, which achieved some success as a result of contact tracing systems [26], the proposed solution has been designed to take into account the privacy of the workers, as highlighted in Section 3.3.

\subsection{Limitations of the study}

First of all, the tests described in this paper were carried out in a simplified environment and considering separately the movement and the related real-time detection of the resources "workers" and "equipment" (i.e., construction machineries). In fact, the tests do not fully consider the complexities linked to the dynamism of a construction site.

As regards the technologies adopted, which have been selected for their easy availability on the market and low cost that has enabled them to be used effectively within the time and economic resources of this project, the problem of antenna direction and orientation of UWB tags and anchors to ensure continuous signal transmission regardless of the positioning and movement of the resources has not yet been resolved.

A further limitation of this project is that, to date, no indications have been given as to the actual distances to be maintained between the operator and the moving construction machinery in order to successfully guarantee the safety of workers in the event of the risk of being run over or hit by moving construction machineries. These analyses are in the development phase and need to take into account all the aspects related to the quality of the data as well as the time needed for its recording, processing and subsequent communication in the PWS.

\subsection{Future works}

Future works will validate the measurement system in the context of actual construction sites, with a focus on a panel of construction activities that requires collaboration between workers and construction machineries (e.g., procedure of excavation).

Moreover, a further series of tests will be carried out that integrate the system architecture with certified telemetry systems in order to set it up a correct monitoring process and assess the effective validity of the digital twin represented in the virtual environment.

A suitable notification system will be set up in order to communicate the presence of a safety risk to the workers, once the PWS detects excessive proximity between workers and moving construction machineries. These notification systems will have to consider the conditions of disturbance and noise typical of construction sites as well as the risk of a decrease in the attention of operators in the event of receiving excessive notifications.

Furthermore, in order to optimise the PWS in terms of technological equipment, a collaboration with manufacturers could allow acting directly on the construction machinery itself, hypothesising scenarios of gradual slowing down and stopping in the event of danger or sending timely notifications and warning signals directly from the equipment itself.

Finally, from the construction management perspective, future developments could also integrate the assessment of the positioning of UWB anchors in the design of construction site layouts and the planning of construction phases so as to include their installation and possible displacement among the activities to be taken into account in order to ensure signal coverage that is always consistent with the activities currently underway.

Generally speaking, the current and expected outcomes show how the proposed and tested technical solutions could be exploited with a broader digital ecosystem, enabling a data-driven control room for safety management.

\section{References}

[1] Zhou, Z., Goh, Y.M., Li, Q. (2015). Overview and analysis of safety management studies in the construction industry. Safety Science, 72, 337-350.

[2] Messi, L., Naticchia, B., Carbonari, A., Ridolfi, L., Di Giuda, G. M. (2020). Development of a Digital Twin Model for Real-Time Assessment of Collision Hazards. In Proc. of Creative Construction $e$ Conference 2020, 14-19.

[3] US BLS (2020). Number and rate of fatal work injuries, by industry sector. Available online: https://www.bls.gov/charts/census-of-fataloccupational-injuries/number-and-rate-of-fatalwork-injuries-by-industry.htm. Last access: 30/09/2021.

[4] INAIL (2019). Dati INAIL: Andamento degli infortuni sul lavoro e delle malattie professionali, 9, ISSN 2035-5645.

[5] Teizer, J., Allread, B., Fullerton, C., Hinze, J. (2010). Autonomous Pro-Active Real-time Construction Worker and Equipment Operator Proximity Safety Alert System. Automation in Construction, 19, 630-640.

[6] Guglielmi, A., Olori, M., Piga, G., Campo, G., Vidale, F. (2017). Investimento dei lavoratori in ambienti di lavoro, Sistema di sorveglianza degli infortuni mortali sul lavoro, Scheda 8, INAIL, 
ISBN 978-88-7484-540-8.

[7] Hosny, A., Nik-Bakht, M., Moselhi, O. (2020). Workspace planning in construction: nondeterministic factors. Automation in Construction 116.

[8] Messi, L., Vaccarini, M., Carbonari, A., Corneli, A., Naticchia, B. (2021). Technology framework for real-time assessment of spatial conflicts in building retrofitting. In Proc. of 2021 European Conference on Computing in Construction.

[9] Jeelani, I., Albert, A., Han, K., Azevedo, R. (2019). Are visual search patterns predictive of hazard recognition performance? Empirical investigation using eye-tracking technology, Journal of construction engineering and management, 145(1).

[10] Bahn, S. (2013). Workplace hazard identification and management: the case of an underground mining operation, Safety science, 57, 129-137.

[11] Chan, K., Louis, J., Albert, A. (2020). Incorporating worker awareness in the generation of hazard proximity warnings, Sensors, 20(3), 806.

[12] Xiao, B., Kang, S. C. (2021). Vision-based method integrating deep learning detection for tracking multiple construction machines, Journal of Computing in Civil Engineering, 35(2).

[13] Awolusi, I., Marks, E., Hallowell, M. (2018). Wearable technology for personalized construction safety monitoring and trending: review of applicable devices, Automation in construction, 85, 96-106.

[14] Park, J., Kim, K., Cho, Y.K. (2016). Framework of automated construction-safety monitoring using cloud-enabled BIM and BLE mobile tracking sensors, Journal of Construction Engineering and Management, 143(2).

[15] Lee, H.-S.; Lee, K.-P.; Park, M.; Baek, Y.; Lee, S. (2011). RFID-based real-time locating system for construction safety management, Journal of Computing in Civil Engineering, 26(3), 366-377.

[16] Amicucci, G., Fiamingo, F. (2016). RFId (RadioFrequency Identification) in applicazioni di sicurezza, INAIL: Dipartimento innovazioni tecnologiche e sicurezza degli impianti, prodotti e insediamenti antropici, ISBN 978-88-7484-524-8.

[17] Ferrari, P., Rinaldi, S., Sisinni, E., Colombo, F., Ghelfi, F., Maffei, D., Malara, M. (2019). Performance evaluation of full-cloud and edgecloud architectures for Industrial IoT anomaly detection based on deep learning. In Proc. of IEEE International Workshop on Metrology for Industry 4.0 and IoT, MetroInd 4.0 and IoT 2019, 420-425.

[18] Ferrari, P., Sisinni, E., Depari, A., Flammini, A., Rinaldi, S., Bellagente, P., Pasetti, M. (2020). On the performance of cloud services and databases for industrial IoT scalable applications, Electronics
(Switzerland), 9 (9), 1-17.

[19] Bellagente, P., Bonafini, F., Crema, C., Depari, A., Ferrari, P., Flammini, A., Sisinni, E. (2018). Enhancing access to industrial IoT measurements by means of location based services. In IEEE Instrumentation \& Measurement Magazine, 21(6), 15-21.

[20] Patel, K., Massa, K., Raghunathan, N., Zhang, H., Iyer, A., Bagchi, S. (2020). Proactive privacypreserving proximity prevention through bluetooth transceivers. In Proc. of the 18th ACM Conference on Embedded Networked Sensor Systems, 778-779.

[21] Baek, J., Choi, Y. (2020). Smart glasses-based personnel proximity warning system for improving pedestrian safety in construction and mining sites, International Journal of Environmental Research and Public Health, 17 (4).

[22] Kim, Y., Baek, J., Choi, Y. (2021). Kim, Y., Baek, J., \& Choi, Y. (2021). Smart Helmet-Based Personnel Proximity Warning System for Improving Underground Mine Safety, Applied Sciences, 11(10).

[23] Mgbemena, C. E., Onuoha, D. O., Okpala, C. C., Mgbemena, C. O. (2020). Design and development of a proximity warning system for improved safety on the manufacturing shop floor, Journal of King Saud University-Engineering Sciences.

[24] Bonafini, F., Depari, A., Ferrari, P., Flammini, A., Pasetti, M., Rinaldi, S., Sisinni, E., Gidlund, M. (2019). Exploiting localization systems for LoRaWAN transmission scheduling in industrial applications. In Proc. of IEEE International Workshop on Factory Communication Systems.

[25] Decawave (2021). Sources of error in dw1000 based two-way ranging (twr) schemes, APS011 Application note. Available online : https://www.decawave.com/wpcontent/uploads/2018/10/APS011_Sources-ofError-in-Two-Way-Ranging-Schemes_v1.1.pdf. Last access: 30/09/2021.

[26] Vecchia, D., Corbalán, P., Istomin, T., Picco, G. P. (2019). TALLA: Large-scale TDoA localization with ultra-wideband radios. In Proc. of International Conference on Indoor Positioning and Indoor Navigation (IPIN), 1-8.

[27] Nguyen, T.D., Miettinen, M., Sadeghi, A.R. (2020). Long live randomization: on privacy-preserving contact tracing in pandemic. In Proc. of the 7th ACM Workshop on Moving Target Defense, 1-9.

\section{Acnowledgements}

The research project is developed in collaboration with Ente Sistema Edilizia Brescia (ESEB) and it is funded by Lombardy Region and Chambers of Commerce Brescia. 